\title{
LÁCTEOS BOVINOS Y PERCEPCIÓN DE LA LECHE CAPRINA ENTRE ESTUDIANTES DE LA UNIVERSIDAD DE COSTA RICA ${ }^{1}$
}

\author{
Pedro Vargas-Aguilar ${ }^{2}$, María Lourdes Pineda-Castro ${ }^{2}$,Alejandro Chacón-Villalobos ${ }^{3}$
}

\begin{abstract}
RESUMEN
Lácteos bovinos y percepción de la leche caprina entre estudiantes de la Universidad de Costa Rica. Se pretendió determinar diferencias significativas entre las características edad, género, práctica de ejercicio físico y hábitos de consumo de lácteos, además de sus interrelaciones en los estudiantes de la Universidad de Costa Rica. Se indagó el conocimiento y la percepción que éstos tienen de la leche de cabra. En septiembre de 2005 se efectuó un estudio básico del perfil del consumidor de lácteos empleando una metodología de encuesta aleatoria en 506 estudiantes ( 272 mujeres y 234 hombres entre 17 y 26 años) evaluando edad, género, práctica de ejercicio, hábitos de consumo, producto más consumido, características de aceptación e impresión sobre la leche de cabra. Los datos codificados se evaluaron con una prueba de chi cuadrado. La población presentó edades oscilantes en un $90 \%$ en el intervalo de 18 a 24 años. La leche $(38,1 \%)$, queso $(14,2 \%)$ y yogur $(9,9 \%)$ fueron en su orden los productos más consumidos, siendo adquiridos preferentemente en supermercados (59,5\%). Un 64,5\% de la población consume diariamente lácteos, representando la ingesta ocasional un $28,3 \%$ y la nula un $5,9 \%$, lo que sugiere deficiencias en la ingesta de calcio. Las mujeres evidencian ser más propensas a buscar productos descremados si no practican ejercicios. Un buen sabor resulta ser innegablemente la característica más demandada $(50,4 \%)$ en los productos. Como es característico en el país, otras fuentes alternativas de leche y productos lácteos como la leche de cabra no están muy difundidas en la población estudiantil, la cual refleja un bajo consumo y un pobre conocimiento de dichas fuentes.
\end{abstract}

Palabras clave: Leche de cabra, leche de vaca, consumo, hábitos nutricionales, universitarios.

\begin{abstract}
Bovine dairy and goat milk perceptions among students of the University of Costa Rica. Our objective was to establish meaningful differences between age, gender, exercise practice and consumption habits and its interactions among students of the University of Costa Rica. The knowledge and the perception of goat milk among these persons were studied as well. In September 2005 a study devoted to understand consumer habits related to dairy products took place among 506 students (272 women and 234 men between 17 and 26 years) using the random interview technique to profile age, gender, exercise, most consumed products, quality demanded and overall knowledge about goat milk. Coded data were evaluated using the chi square test. The population age ranged $90 \%$ between 18 and 24 years. Milk (38.1\%), cheese (14.2\%) and yogurt $(9.9 \%)$ were the most consumed products, which are usually bought at supermarkets (59.5\%). About $64.5 \%$ of the population consumes dairy products on a daily basis but $28.3 \%$ only have these products occasionally and $5.9 \%$ almost never. Such nutritional behavior could be linked with a poor calcium intake. Women preferred low-fat products, especially if they are not involved in some exercise practice. Good flavor is undeniably the most important characteristic demanded by dairy consumers $(50.4 \%)$. As it is usual in Costa Rica, non traditional sources of milk such as goat milk and related products are not very popular among the students, fact that can be noted in their low consumption and the poor knowledge when it comes to this milk.
\end{abstract}

Key words: Goat milk, cow milk, consumption, nutritional habits, universitarians.

\footnotetext{
1 Recibido: 30 de marzo, 2006. Aceptado: 5 de marzo, 2007. Proyecto inscrito en Vicerrectoría de Investigación No.737-A4-040, Universidad de Costa Rica.

2 Escuela de Tecnología de Alimentos, Universidad de Costa Rica. Correo electrónico: pvargas@ cariari.ucr.ac.cr; mlpineda@cariari.ucr.ac.cr

3 Estación Experimental Alfredo Volio, Universidad de Costa Rica. Correo electrónico: achaconv@cariari.ucr.ac.cr
} 


\section{INTRODUCCIÓN}

La producción de lácteos es una de las agroindustrias más pujantes de Costa Rica, tanto a nivel agrosilvopastoril como agroindustrial (Díaz 2004). El auge de esta actividad está acompañado por una tasa de crecimiento en el consumo de lácteos que supera el aumento anual de la población (Vega 2002). La ingesta de leche y sus derivados en Costa Rica y su aporte proteico son de los más altos entre los países de América Latina (Morón et al. 2005). No obstante, para Latinoamérica se considera que el consumo per capita de lácteos está por debajo de las recomendaciones internacionales (Espíndola 2004).

El consumo de leche y sus derivados se ha asociado con una dieta de calidad, que proporciona la ingesta adecuada de muchos nutrientes, incluyendo calcio, potasio, magnesio, zinc, hierro, riboflavina, vitamina A, folato y vitamina D (USDA 2005). En este sentido, Severi y Girona (2005) señalan el consumo de calcio y la actividad física como los principales aliados en la formación saludable de los huesos, constituyendo los lácteos en sí mismos la principal fuente de calcio de la dieta (Chacón 2005). Esto ha llevado al establecimiento de requerimientos mínimos de productos lácteos, según edad, sexo y estado fisiológico de las personas.

Los principales factores asociados al consumo de productos lácteos son: la edad, el nivel socio económico, el nivel académico, la accesibilidad (compra o programas alimentarios o sociales), la disponibilidad, el género y los elementos socioculturales (hábitos alimentarios, educación, conveniencia, rutinas, estilos de vida) (Severi y Girona 2005; Carriquiry 2005).

Varios estudios internacionales (Girona y Severi 2004), han puesto en evidencia que en general la ingesta de calcio durante la infancia y la adolescencia no siempre es la adecuada, y que además los adolescentes son un grupo de riesgo no sólo por los grandes cambios biológicos, sicológicos y sociales que enfrentan, sino por los hábitos alimentarios que suelen practicar. Una de las razones por la cual este grupo en particular tiende a marginar el consumo de lácteos, es la creencia de que la leche y sus derivados engordan (Fernández 2004).
En Costa Rica son los lácteos de origen bovino los que se consumen mayoritariamente (Corrales y Chacón 2005; Rojas 2005). Otras fuentes alternativas como la leche de cabra parecen tener una baja aceptación a nivel del mercado general, debido quizás a aspectos relacionados con ciertos prejuicios sensoriales y hasta culturales (Corrales y Chacón 2005). No obstante esta última representa un mercado potencial de factible surgimiento en segmentos poblacionales interesados en una dieta saludable, dado el sinfín de cualidades nutricionales y hasta nutracéuticas científicamente documentadas que la misma presenta (Chacón 2005). Los jóvenes universitarios podrían representar un segmento con tales características, razón por la cual paralelamente al estudio de los hábitos de consumo de la leche bovina y sus derivados, se decidió sondear aspectos relacionados con la percepción de la leche de cabra en este nicho específico con la finalidad de establecer algunos antecedentes de utilidad futura.

Por lo anteriormente expuesto, Severi y Girona (2005) afirman que es necesario desarrollar investigaciones que colaboren a la comprensión de los hábitos de consumo de los lácteos, de modo que sus resultados se traduzcan en estrategias efectivas de promoción de los mismos que aborden integralmente aspectos como las cantidades consumidas, tiempos, formas de preparación, valor asignado al alimento, etc. Por esta razón, el presente estudio pretendió determinar si existen diferencias estadísticamente significativas entre las características (edad, género y práctica de ejercicio físico) y sus interrelaciones y los hábitos de consumo de lácteos (como producto más consumido, frecuencia de consumo, lugar de compra) y sus interrelaciones en los estudiantes de la Universidad de Costa Rica. Además, se indagó el conocimiento y la percepción que tienen los estudiantes universitarios con respecto a la leche de cabra.

\section{MATERIALES Y MÉTODOS}

El estudio se efectuó durante el mes de septiembre del año 2005, en el campus de la Ciudad Universitaria Rodrigo Facio de la Universidad de Costa Rica ubicado en la provincia de San José. El mismo consistió en un estudio básico del perfil del consumidor de 
lácteos en 506 estudiantes de grado, pertenecientes a la población universitaria regular (272 mujeres y 234 hombres), con un rango de edades comprendido entre 17 y 26 años. Se diseñó con este fin, un formulario de encuesta según las recomendaciones de Corrales y Chacón (2005), Rojas (2005) y de Ivankovich $(2003)^{4}$. La encuesta incluyó preguntas cerradas para caracterizar la población en cuanto a edad, género, práctica de ejercicio físico, y hábitos de consumo de productos lácteos (frecuencia y lugar de compra). Se incluyeron preguntas abiertas adicionales para poder conocer el producto lácteo más consumido por cada persona y la característica principal de aceptación que busca en estos productos. Adicionalmente, se hicieron preguntas abiertas sobre el conocimiento y opinión en general, sobre la leche de cabra, producto que en la actualidad está recibiendo mucha atención debido a sus propiedades nutricionales (Chacón 2005).

La encuesta fue aplicada en forma aleatoria a la población en estudio, entrevistando a los pasantes simultáneamente en cinco zonas diferentes y concurridas, que abarcaban todo el campus, para una mayor representatividad. Se siguió la metodología de entrevista.

Los datos recopilados fueron codificados para cada pregunta tabulando en cuadros de frecuencia las respuestas para su evaluación estadística. Utilizando el programa JMP-SAS versión 4.0 se realizó la prueba de chi cuadrado $\left(\mathrm{X}^{2}\right)$ para valorar la relación entre las variables categóricas. Con la ayuda de los gráficos y los cuadros de contingencia elaborados por el programa, se establecieron los principales efectos que explican las relaciones encontradas, según lo sugerido por O'Mahony (1986).

\section{RESULTADOS Y DISCUSIÓN}

La Figura 1 muestra la distribución de la población evaluada sobre la base del parámetro edad. El $90,0 \%$ de los universitarios encuestados tuvo una edad que varió entre los 18 y 24 años.

\footnotetext{
4 IVANKOVICH, C. 2003. Sondeo de mercado con consumidores habituales. Centro Nacional de Ciencia y Tecnología de Alimentos. San José. Costa Rica. Comunicación personal.
}

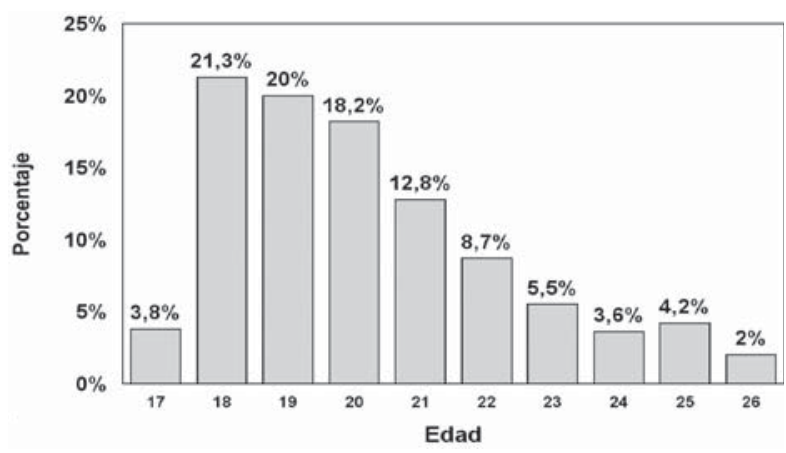

Figura 1. Distribución por edades de la población evaluada. San José, Costa Rica. 2005.

Se encontró que un $1 \%$ del alumnado total no consume productos lácteos de ninguna naturaleza, resultados que son similares a los obtenidos en España (Ministerio Español De Agricultura, Pesca y Alimentación 2004b), donde el 0,6\% de los encuestados nunca consumen productos lácteos. En general, se considera que la mayoría pertenece a un grupo de personas que no ingieren estos productos por razones especiales como pueden ser las alergias, la intolerancia, el sabor u otras razones como las prácticas culturales y hasta religiosas (Chacón 2005; USDA 2005).

La leche resulta ser el producto más consumido por los universitarios (Cuadro 1), independiente del género o si se practica algún ejercicio.

En España para la población general, el gasto en leche líquida es un $4,4 \%$ del gasto total per cápita en alimentación, lo que representa un 36,7\% del total de gasto en productos lácteos (Ministerio Español De Agricultura, Pesca y Alimentación, 2004a). Lo anterior indica que en este país el principal producto lácteo consumido es la leche, lo cual es evidente en los informes de la Federación Nacional de Industrias Lácteas de España (2005). También en Argentina el consumo per cápita de leche fluida $(40,38 \mathrm{~kg} / \mathrm{hab} / \mathrm{año})$ reportado para el año 2004 supera ampliamente al de otros productos lácteos como el yogurt $(9,35 \mathrm{~kg} / \mathrm{hab} / \mathrm{año}) \mathrm{o}$ el queso $(9,01 \mathrm{~kg} / \mathrm{hab} / \mathrm{año})$ Secretaria de Agricultura, Ganadería. Pesca y Alimentos de La República Argentina 2005). En el caso particular de Costa Rica, Severi y Girona (2005) reportaron un consumo diario medio de $529 \mathrm{~g}$ de leche para el año $2001 \mathrm{y}$, según Vega 
Cuadro 1. Consumo de productos lácteos en la población evaluada según la realización de ejercicio y el género. San José, Costa Rica. 2005.

\begin{tabular}{|c|c|c|c|c|c|c|c|c|c|}
\hline \multirow{2}{*}{ Producto preferido } & \multicolumn{3}{|c|}{ Realiza ejercicio } & \multicolumn{3}{|c|}{ No realiza ejercicio } & \multirow{2}{*}{$\begin{array}{l}\text { Total de } \\
\text { hombres }\end{array}$} & \multirow{2}{*}{$\begin{array}{l}\text { Total de } \\
\text { mujeres }\end{array}$} & \multirow[t]{2}{*}{ Total } \\
\hline & Hombre & Mujer & Total & Hombre & Mujer & Total & & & \\
\hline Leche & 79 & 37 & 116 & 35 & 42 & 77 & 114 & 79 & 193 \\
\hline Queso & 14 & 23 & 37 & 12 & 23 & 35 & 26 & 46 & 72 \\
\hline Yogurt & 7 & 16 & 23 & 4 & 23 & 27 & 11 & 39 & 50 \\
\hline Leche y queso & 8 & 14 & 22 & 7 & 5 & 12 & 15 & 19 & 34 \\
\hline Leche, queso y yogurt & 10 & 10 & 20 & 1 & 3 & 4 & 11 & 13 & 24 \\
\hline Leche y yogurt & 4 & 3 & 7 & 2 & 8 & 10 & 6 & 11 & 17 \\
\hline Mantequilla & 1 & 4 & 5 & 0 & 8 & 8 & 1 & 12 & 13 \\
\hline Helado & 2 & 2 & 4 & 1 & 8 & 9 & 3 & 10 & 13 \\
\hline Natilla & 2 & 6 & 8 & 2 & 2 & 4 & 4 & 8 & 12 \\
\hline No responde & 28 & 13 & 41 & 15 & 22 & 37 & 43 & 35 & 78 \\
\hline Totales & 155 & 128 & 283 & 79 & 144 & 223 & 234 & 272 & 506 \\
\hline
\end{tabular}

(2002), para ese mismo año se estimó un consumo per cápita anual de lácteos de 192,2 kg ELF5. Este consumo per capita estaría compuesto por 35\% de queso, $27,5 \%$ de leche fluida y un $19,1 \%$ de natilla y yogurt. Considerando los estudios citados anteriormente y los resultados de esta investigación, se hace evidente que la leche es en sí misma un producto lácteo que tiende a ser el más demandado en la población, aspecto que se refleja también en un segmento tan específico como el de los estudiantes universitarios.

El segundo producto de mayor consumo por los universitarios es el queso. Según un estudio realizado por Ivankovich et al. (1990), para la población de Costa Rica en general, los factores que impulsan la compra de queso son el sabor, la funcionalidad y la variedad. El mismo estudio señala que en el $73,7 \%$ de los hogares costarricenses de todos los estratos sociales, se encuentra el queso fresco como parte de las compras regulares de la familia promedio. Otros tipos de quesos como el queso crema (34,5\% de los hogares) y el queso rallado $(14,9 \%)$ son también populares. A partir de lo expuesto anteriormente, es posible argumentar que siendo el queso un producto generalmente de alta disponibilidad en los hogares costarricenses, no es de extrañar que un porcentaje importante de los universitarios lo consuma habitualmente desde etapas tan tempranas como la niñez, y que, por lo tanto, los resultados obtenidos puedan ser un reflejo de estos hábitos.

5 ELF: unidades equivalentes de leche fluída.
La población evaluada se caracterizó seguidamente para las variables género y ejercicio físico. Un análisis logístico indica que existe una relación estadísticamente significativa $\left(X^{2}=18,95 ; p<0,001\right)$ entre practicar ejercicio y el género. Tal y como lo manifiesta la Figura 2, una proporción mayor de hombres que de mujeres reporta la práctica de algún tipo de ejercicio físico.

La prueba de chi cuadrado realizada a los datos demuestra que existe una diferencia significativa en el producto preferido por los universitarios según el género $\left(X^{2}=45,04 ; p<0,001\right)$ o si realizan o no ejercicio $\left(X^{2}=20,58 ; p<0,05\right)$. Para encontrar exactamente el patrón de comportamiento de la distribución se deben inspeccionar los datos, según recomienda O'Mahony (1986).

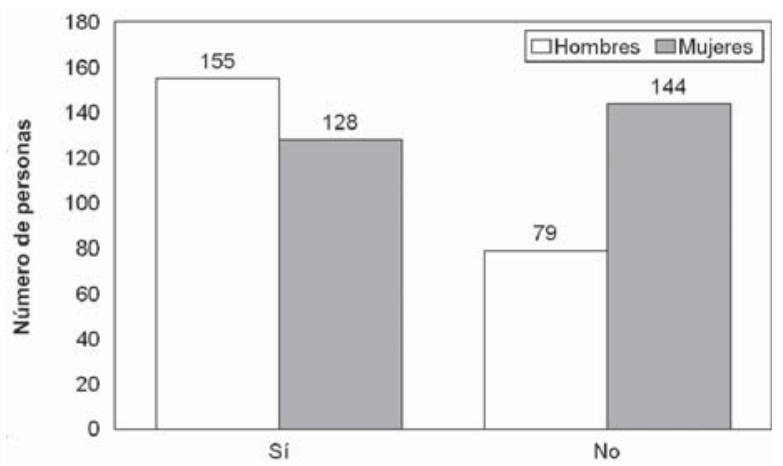

Figura 2. Distribución de la realización de ejercicio físico en la población evaluada. San José, Costa Rica. 2005. 
La leche es consumida preferiblemente por encima de otros productos lácteos en una proporción mayor de hombres (49\%) que de mujeres (29\%). Por otra parte, una mayor proporción de quienes hacen ejercicio $(55 \%)$ consumen la leche como principal producto lácteo, en comparación con aquellos que no hacen ejercicio (44\%).

Con respecto al consumo del yogurt, el helado y la mantequilla, se debe destacar que es mayor en el grupo de mujeres que no hacen ejercicio. El consumo de combinaciones de queso con otros productos lácteos es mayor en los estudiantes de ambos géneros que realizan ejercicio.

En el Cuadro 2 se presenta la distribución de la población en función de los atributos de aceptación más importante que los estudiantes buscan en un producto lácteo. El sabor es la principal característica demandada, seguida de la frescura y la textura. El análisis logístico de los datos indica que el ejercicio influye significativamente $\left(\mathrm{X}^{2}=15,17 ; \mathrm{p}<0,05\right)$ sobre los atributos deseados en los productos lácteos, no así el género $\left(X^{2}=6,5 ; p>0,05\right)$.

Cuadro 2. Atributos más buscados en los productos lácteos según la práctica de ejercicio físico. San José, Costa Rica. 2005.

\begin{tabular}{lrcr}
\hline Atributo & $\begin{array}{c}\text { Realiza } \\
\text { ejercicio }\end{array}$ & $\begin{array}{c}\text { No realiza } \\
\text { ejercicio }\end{array}$ & Total \\
\hline Sabor & 156 & 99 & 255 \\
Frescura & 16 & 15 & 31 \\
Textura & 14 & 13 & 27 \\
Nutrición & 8 & 14 & 22 \\
Descremado & 5 & 15 & 20 \\
Presentación & 6 & 5 & 11 \\
Otras & 44 & 31 & 75 \\
No responde & 34 & 31 & 65 \\
Total & 283 & 223 & 506 \\
\hline
\end{tabular}

Otras características adicionales a las listadas en el Cuadro 2 fueron obtenidas debido a que la pregunta correspondiente en los formularios aplicados fue con respuesta abierta, y las personas tendieron en muchos casos a dar respuestas con apreciaciones personales de la más variada índole pero que no son significativas dentro del total de respuestas recopiladas. Algunas de estas respuestas misceláneas señalaron parámetros apreciados tales como el precio, el color, el aroma, la marca comercial, el estado de conservación del producto, la capacidad de derretirse al calor con facilidad (posiblemente refiriéndose al queso), la accesibilidad (que siempre hayan existencias de producto en el lugar donde se compra habitualmente) y la higiene en el punto de venta.

El $55 \%$ de los que practican algún ejercicio consideran el sabor como la característica principal requerida para que un producto sea aceptable, en tanto que esta característica es la más importante para solamente el $44 \%$ de quienes no hacen ejercicio. No se logró encontrar literatura que secunde las relaciones halladas en el presente estudio entre el ejercicio y la predilección por el sabor como principal atributo de la leche y los productos lácteos.

Por otro lado, de los estudiantes entrevistados que se preocupan por los aspectos nutricionales de los productos lácteos, el $64 \%$ no hace ejercicio. Aunque el género no resultó estadísticamente significativo sobre los atributos preferidos en los productos lácteos, es importante señalar que, entre aquellos estudiantes para los que es importante que el producto sea descremado, el $60 \%$ son mujeres que no hacen ejercicio. Es posible que estas mujeres, al estar concientes de su inactividad física y dada la presión social usual, prevengan más un posible aumento de peso demandando productos descremados. Además, Davy et al. (2006), quienes hicieron un estudio con estudiantes universitarios, afirman que más mujeres que hombres consumen diariamente dietas bajas en grasas y carbohidratos debido a que generalmente tienen mayor conocimiento sobre aspectos de nutrición, dada la necesidad de perder o mantener peso.

Se puede notar en el Cuadro 2 que quienes se ejercitan, están menos preocupados por los aspectos nutricionales del producto tales como el que éste sea descremado por ejemplo, probablemente por la sensación de "salud y control del peso" derivadas de hacer más ejercicio, siendo, bajo estas circunstancias que las características sensoriales como el sabor son más apreciadas. 
Existió una relación significativa $\left(\mathrm{X}^{2}=125,18\right.$; $\mathrm{p}<0,001)$ entre las características deseadas en los productos lácteos y la preferencia por un determinado producto. Para la leche y los demás derivados lácteos, el sabor fue el atributo más importante en la población universitaria (Cuadro 3). Además en la leche, el queso y el yogurt los consumidores le dan importancia adicional a otros atributos como la frescura, la textura, el valor nutricional y el descremado. Todos los demás atributos varían de acuerdo al producto, sin notarse claramente que alguno sea relevante, tal y como se observa en el Cuadro 3.

En la Figura 3 se observa que un 64,8\% de los estudiantes consumen lácteos todos los días, aunque no se valoró si ingieren un número de porciones equivalente a las recomendadas. Estudios efectuados en otros países, como es el caso de España, han determinado que el $88,1 \%$ de los consumidores admite consumir productos lácteos todos o casi todos los días, lo cual guarda una moderada similitud con el $64,8 \%$ observado en los universitarios de Costa Rica (Ministerio Español De Agricultura, Pesca y Alimentación 2004b). Es obvio con base en los datos obtenidos que al menos un $35,2 \%$ de los estudiantes costarricenses no satisfacen los requerimientos de consumo de productos lácteos, ya que no los consumen todos los días. Gerometta et al. (2004) entrevistaron en España a un grupo de estudiantes universitarios de ambos sexos resultando que el 7,6\% afirmó no consumir lácteos nunca, cifra que presenta un cierto paralelismo con el 5,9\% encontrado en sus pares costarricenses (Figura 3).

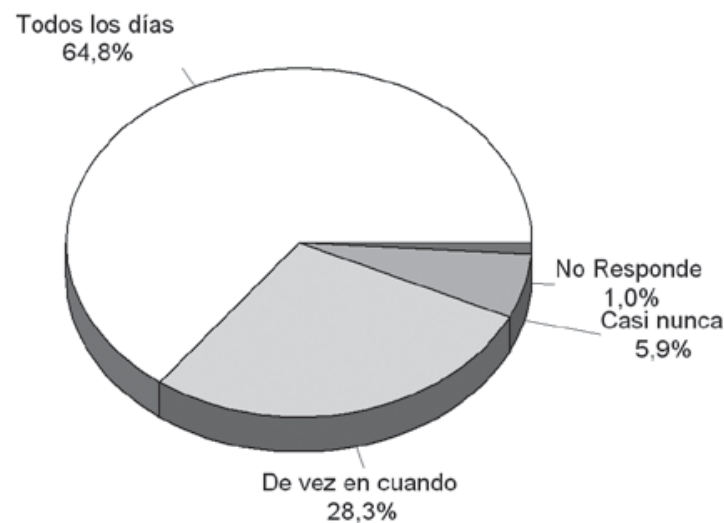

Figura 3. Frecuencia de consumo de productos lácteos entre los universitarios encuestados. San José, Costa Rica. 2005.

La escasa ingesta de lácteos en algunos estudiantes evidenciada en este caso, implica que no garantiza el requerimiento de ingesta recomendado, correspondiente a tres tazas de leche o tres porciones de productos lácteos para la población en general (USDA 2005) y también para los adolescentes entre 14 y 18 años (Gidding et al. 2005). El consumo de las cantidades

Cuadro 3. Distribución de la población universitaria según los atributos considerados más importantes en cada uno de los productos lácteos. San José, Costa Rica. 2005.

\begin{tabular}{|c|c|c|c|c|c|c|c|c|c|}
\hline & Sabor & Frescura & Textura & Nutrición & Descremado & Presentación & Otras & No responde & Total \\
\hline Leche & 89 & 14 & 6 & 13 & 8 & 1 & 26 & 36 & 193 \\
\hline Queso & 45 & 4 & 5 & 0 & 0 & 3 & 8 & 7 & 72 \\
\hline Yogurt & 25 & 0 & 9 & 2 & 0 & 0 & 10 & 4 & 50 \\
\hline Leche y queso & 23 & 2 & 3 & 1 & 1 & 0 & 3 & 1 & 34 \\
\hline \multicolumn{10}{|l|}{ Leche, queso y } \\
\hline yogurt & 16 & 2 & 0 & 1 & 0 & 3 & 1 & 1 & 24 \\
\hline Leche y yogurt & 9 & 2 & 0 & 0 & 0 & 0 & 2 & 4 & 17 \\
\hline Mantequilla & 7 & 0 & 1 & 0 & 1 & 0 & 4 & 0 & 13 \\
\hline Helado & 5 & 0 & 1 & 0 & 0 & 1 & 3 & 3 & 13 \\
\hline Natilla & 7 & 0 & 1 & 0 & 1 & 0 & 1 & 2 & 12 \\
\hline No Responde & 29 & 7 & 1 & 5 & 9 & 3 & 17 & 7 & 78 \\
\hline Total & 255 & 31 & 27 & 22 & 20 & 11 & 75 & 65 & 506 \\
\hline
\end{tabular}


recomendadas de productos lácteos es muy importante porque, al menos en la dieta de los habitantes de los Estados Unidos, representa un $70 \%$ de la ingesta de calcio (USDA 2005). Girona y Severi (2004), en su estudio realizado en Costa Rica para estimar el consumo dietético de calcio entre los adolescentes urbanos y rurales de 13-18 años, demuestran que el $82 \%$ de los adolescentes no alcanzan en la ingesta los dos tercios de las recomendaciones nutricionales de calcio. Una proporción significativamente mayor de mujeres (91\%) que de hombres $(77 \%)$ evidenció a la vez un consumo marginal de calcio. En un trabajo de Aldabe y Márquez (2005) realizado en Uruguay con niños en edad escolar, se detectó que una cierta proporción no alcanza a ingerir las cantidades recomendadas de lácteos para lograr el adecuado aporte de calcio y que esta proporción se hace mayor a partir de los 11 años. Esto se debe a las reducidas cantidades consumidas y a la inadecuada frecuencia en que se realiza dicha ingesta.

Esto demuestra que los productos lácteos no están cumpliendo el papel en la nutrición de los adolescentes y adultos jóvenes, en cuanto al aporte de nutrientes esenciales como calcio y proteína de alta calidad, que se espera de este grupo de alimentos.

Ni el género $\left(X^{2}=4,03 ; p>0,05\right)$ ni la actividad física $\left(X^{2}=2,62 ; p>0,05\right)$ tuvieron efecto significativo sobre la frecuencia de consumo de lácteos en los estudiantes universitarios entrevistados en el presente estudio. Sin embargo, los datos parecen sugerir que las personas que realizan ejercicio tienen una tendencia a consumir productos lácteos más frecuentemente. Esto último podría estar asociado con el ya mencionado temor de las personas más sedentarias a sufrir un aumento de peso derivado del consumo de productos lácteos (Fernández 2004). Adicionalmente, cuando se hace ejercicio, el calcio que se requiere para la contracción muscular se extrae de los huesos. Si una persona se ejercita regularmente y no cubre la demanda de calcio, corre el riesgo de descalcificarse (Weaver 2000; Forslund et al. 2000). Por esta razón, resulta altamente positivo que las personas que realizan algún tipo de ejercicio físico presenten un consumo más alto de productos lácteos, sea este consciente o inconsciente.

En la Figura 4 se muestra como el 59,5\% de los consumidores universitarios adquieren sus productos

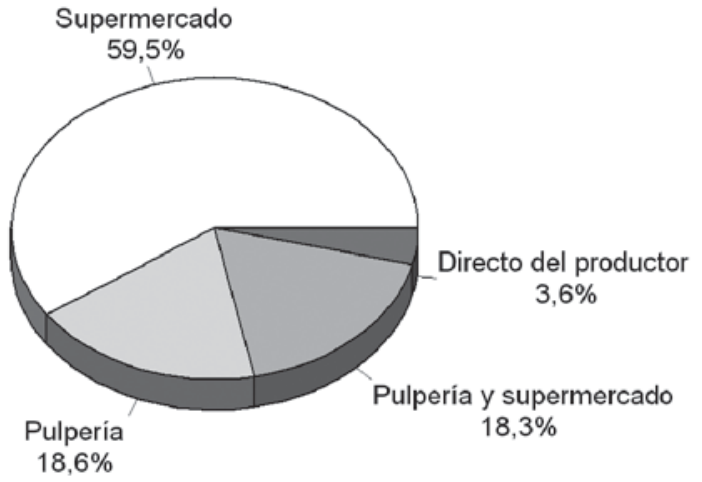

Figura 4. Lugar en que los estudiantes adquieren los productos lácteos. (Pulperías: Pequeños y medianos locales de venta al detalle de abarrotes muy populares en los suburbios y otras áreas rurales de Costa Rica). San José, Costa Rica. 2005.

preferentemente en el supermercado, mientras que solamente el $3,5 \%$ lo compra directamente al productor. Para el mercado costarricense muchos de los productores que hacen venta directa son artesanales (Díaz 2004). Adicionalmente se ha señalado a la proximidad, la calidad de los productos y los buenos precios, como los principales factores que deciden la elección de un determinado establecimiento para la compra de alimentos (Ministerio Español De Agricultura, Pesca y Alimentación 2004b).

A lo largo del presente estudio, se hace evidente que los estudiantes universitarios son consumidores de la leche y de sus productos derivados. No obstante es primordial resaltar que dicho consumo es mayoritariamente de productos elaborados con leche de vaca, la cual es la de mayor tradición en el país (Díaz 2004). Los números no son los mismos cuando se trata de fuentes menos tradicionales, más recientes y más incipientes como es el caso de la leche de cabra. La poca interacción de los costarricenses con los productos obtenidos a partir de la leche de cabra se ve reflejada en el estudio de Corrales y Chacón (2005), quienes encontraron que el $54 \%$ de los costarricenses nunca han probado el queso de cabra.

Los datos obtenidos en el presente estudio muestran para la población universitaria que el $66,5 \%$ de los 
entrevistados han probado al menos una vez en su vida la leche de cabra o sus productos derivados.

Al consultar a los estudiantes si conocían "algo" sobre la leche de cabra (Cuadro 4) se halló que el 40 $\%$ no conocía nada al respecto, mientras que otros la consideraron saludable $(17,0 \%)$, nutritiva $(14,0 \%)$ y deliciosa (12,3\%). Únicamente un 8,1\% de los encuestados la calificó como de sabor desagradable.

Cuadro 4. Distribución de las personas según su percepción y conocimiento de la leche de cabra. San José, Costa Rica. 2005.

\begin{tabular}{lc}
\hline \multicolumn{1}{c}{ Conocimiento } & Porcentaje (\%) \\
\hline Nada & 40,5 \\
Saludable & 17,0 \\
Nutritiva & 14,0 \\
Deliciosa & 12,3 \\
Sabor desagradable & 8,1 \\
Nutritiva y saludable & 2,6 \\
Calidad & 2,2 \\
Cara & 1,4 \\
Engorda & 1,2 \\
Prefiere leche de vaca & 0,8 \\
\hline
\end{tabular}

Los resultados obtenidos reflejan una vez más la ya abrumadora supremacía de la leche de vaca en el país reportada por la bibliografía, por sobre las otras fuentes no tradicionales como la leche de cabra que representa desde lejos la segunda fuente en importancia (Díaz 2004; Corrales y Chacón 2005; Rojas 2005).

Los resultados obtenidos son de especial relevancia dado que éste representa el primer estudio efectuado con estudiantes de nivel universitario en el país en el tópico específico de los hábitos de consumo de lácteos. Por ello brindan información de primera mano en un apartado que cuenta con escasa investigación existente, y que puede ser de utilidad en las estrategias de inserción y mercadeo de productos tanto existentes como emergentes.

\section{CONCLUSIÓN}

La población estudiantil de la Universidad de Costa Rica está constituida primordialmente por jóvenes
( $53 \%$ mujeres y $47 \%$ hombres) cuyas edades varían en un $90 \%$ de los casos en un intervalo entre los 18 a 24 años y quienes consumen en un $99 \%$ productos lácteos de alguna clase.

La leche $(38,1 \%)$, el queso $(14,2 \%)$ y el yogur $(9,9 \%)$ resultan ser en su orden los productos más consumidos de este segmento poblacional, siendo los mismos adquiridos preferentemente en los supermercados $(59,5 \%)$.

Solamente un $64,5 \%$ de la población encuestada consume diariamente los productos mencionados representando el consumo ocasional un 28,3\% y el casi nulo un $5,9 \%$, valores que pueden sugerir deficiencias en la ingesta de calcio. Las mujeres por su parte evidencian ser más propensas a buscar productos descremados si no practican ejercicios.

De los criterios de aceptación de los lácteos un buen sabor resulta ser innegablemente la característica más demandada $(50,4 \%)$.

Como es característico en el país, otras fuentes alternativas de leche y productos lácteos como la leche de cabra no están muy difundidas en la población estudiantil, la cual refleja un bajo consumo ocasional y un pobre conocimiento del tema.

\section{LITERATURA CITADA}

ALDABE, M.I.; MÁRQUEZ, M. 2005. $1^{\circ}$ encuesta nacional de consumo de lácteos en niños de $1^{\circ}, 3^{\circ}$ y $6^{\circ}$ año de las escuelas públicas uruguayas. Programa Panamericano de Fomento de Consumo de Lácteos - Fepale. Revista Electrónica sobre Leche Escolar, $1^{\mathrm{a}}$ edición, octubre 2005. pp.12-23. Consultado 9 feb. 2006. Disponible en: http://www.infoleche.com/fepale/fepale/lechesalud/Revistahtml/ArtANEP/Encuesta ANEP. htm

CARRIQUIRY, M.R. 2005. Las políticas lecheras y su impacto en el consumo de lácteos. Taller Panamericano: Estrategias para incrementar el consumo de lácteos como aporte a la salud (en línea). Punta del Este. 25 de abril del 2005. Consultado 23 mar. 2005. Disponible en: http://www.infoleche.com/fepale/fepale/MLMS/Ponenciastaller/M. Carriquiry-consumoppt[1].ppt 
CHACÓN, A. 2005. Aspectos nutricionales de la leche de cabra y sus variaciones en el proceso agroindustrial. Agronomía Mesoamericana 16(2): 239-252

CORRALES, J.; CHACÓN, A. 2005. Estudio de opinión de consumidores sobre el queso fresco de cabra (Capra hircus) en Costa Rica. Revista de Agricultura Tropical 35: 39-49.

DAVY, S.R.; BENES, B.A.; DRISKELL, J.A. 2006. Sex differences in dieting trends, eating habits, and nutrition beliefs of a group of midwestern college students. Journal of de American Dietetic Association 106(10): 1673-1677.

DÍAZ, C. 2004. Caracterización de la agroindustria láctea en Turrialba. Costa Rica. Revista de Agricultura Tropical 34: 27-39.

ESPÍNDOLA, H.D. 2004. Programa de fomento del consumo de lácteos. FEPALE (en línea). Consultado 23 mar. 2006. Disponible en: http://www.infoleche.com/ fepale/fepale/CONSUMO DE LACTEOS.ppt

FEDERACIÓN NACIONAL DE INDUSTRIAS LÁCTEAS DE ESPAÑA. 2005. Consumo leche y productos lácteos comparativa mayo 2005/2004 (en línea). Consultado 26 ene. 2006. Disponible en: http://www.fenil. org/Sector/.. Documentos Sector Consumo CompAcumUltimoAnoconsumo_comparativa_mayo05-04.pdf

FERNÁNDEZ, M. 2004. Deficiencias de calcio (en línea). Consultado 29 mar. 2006. Disponible en: http://www. consumaseguridad.com/web/es/sociedad_y_consumo /2004/01/12/10288.php

FORSLUND, A.H.; HAMBRÆUS, L.; VAN BEURDEN, H.; HOLMBÄCK, U.; EL-KHOURY, A.E.; HJORTH, G.; OLSSON, R.; STRIDSBERG, M.; WIDE, L.; ÅKERFELDT, T.; REGAN, M.; YOUNG, V.R. 2000. Inverse relationship between protein intake and plasma free amino acids in health men at physical exercise. American Journal of Physiology - Endocrinology and Metabolism 278: 857-867.

GEROMETTA, P.H.; CARRARA, C.; GALARZA, L.J.A.; FEYLING, V. 2004. Frecuencia de consumo de alimentos en ingresantes a la carrera de medicina. Revista de Posgrado de la VIa Cátedra de Medicina, España no.136: 9-13.

GIDDING,S.S.;DENNISON,B.A.;BIRCH,L.L.;DANIELS, S.R.; GILMAN, M.W.; LICHTENSTEIN, A.H.; RATTAY, K.T.; STEINBERGER, J.; STETTLER, N.;
VAN HORN, L. 2005. Dietary recommendations for children and adolescents. A Guide for Practitioners. Circulation 112: 2061-2075.

GIRONA, A.; SEVERI, C. 2004. Los adolescentes, ¿consumen lácteos en cantidad suficiente? Foro Electrónico Panamericano: La Importancia de los Lácteos en la Salud de los Adolescentes. FEPALE / OPS (en línea). 1 al 17 de septiembre de 2004. Consultado 24 marzo de 2006. Disponible en: http://www.infoleche.com/fepale /fepale/lechesalud/ documentos/DOCUMENTO1FOROSALUD ADOLESCENTE Y LÁCTEOS.doc

IVANKOVICH, C.; AGUILAR, F.; FIGUEROA, J. 1990. Hábitos y motivaciones del consumo de queso en Costa Rica. CITA, Universidad de Costa Rica. San José. 28 p.

MINISTERIO ESPAÑOL DE AGRICULTURA, PESCA Y ALIMENTACIÓN. 2004a. Hechos y cifras de la agricultura, la pesca y la alimentación en España. 7a. ed. Secretaría General Técnica. Ministerio de Agricultura, Pesca y Alimentación. Madrid. 142 p.

MINISTERIO ESPAÑOL DE AGRICULTURA, PESCA Y ALIMENTACIÓN. 2004b. Observatorio del consumo y la distribución alimentaria: Estudios sobre la comercialización agroalimentaria en España. Secretaría General de Agricultura y Alimentación/ Dirección General de Industria Alimentaria y Alimentación. Madrid. 30 p.

MORÓN, C.; ALONSO, L.; CROVETTO, M. 2005. Cambios en la estructura del consumo de alimentos y nutrientes de América Latina 1979-1981 a 1999-2001. Organización de las Naciones Unidas para la Agricultura y la Alimentación (FAO), Oficina Regional de la FAO para América Latina y el Caribe. Santiago de Chile. 63 p.

O'MAHONY, M. 1986. Sensory evaluation of food. Statistical methods and procedures. Marcel Dekker, New York.

ROJAS, W. 2005. Evaluación del efecto de diferentes proporciones de leche de vaca y leche de cabra sobre las características químicas, físicas y sensoriales de un yogurt batido de fresa. Tesis Lic. Tecnología de Alimentos. Escuela de Tecnología de Alimentos. Universidad de Costa Rica. San José, Costa Rica. 99 p.

SECRETARIA DE AGRICULTURA, GANADERÍA. PESCA Y ALIMENTOS DE LA REPUBLICA ARGENTINA. 2005. Consumo de lácteos por productos (en 
línea). Consultado 26 de enero de 2006. Disponible en: http://www.alimentosargentinos.gov.ar/0-3/lacteos/ 06_Consumo/Consumo_03.htm

SEVERI, C.; GIRONA, A. 2005. Caracterización del consumo de lácteos en América Latina. Taller Panamericano: Estrategias para incrementar el consumo de lácteos como aporte a la salud (en línea). Punta del Este. 25 de abril del 2005. Consultado 23 mar. 2005. Disponible en: http://www.infoleche.com/fepale/fepale/MLMS /Ponenciastaller/Severi-Caracterización del consumo 2[1].ppt
USDA. 2005. Dietary guidelines for americans 2005. U.S Department of Health and Human Services / U.S. Department of Agriculture. Washington, D.C. 71 p.

VEGA, O. 2002. Desempeño de la ganadería de leche y de la industria de la transformación de productos lácteos en Costa Rica 1999-2001. SEPSA, Área de Estudios Económicos e Información. San José, Costa Rica. 32 p.

WEAVER, C.M. 2000. Calcium requirements of physically active people. American Journal of Clinical Nutrition 72(suppl): 579S-584S. 